\title{
The presence of chronic diseases contributes to the occurrence risk factors for gynecological cancers in Japan
}

\author{
KAZUHIRO OKAMOTO, KEIICHIRO NAKAMURA, HIROFUMI MATSUOKA, \\ YUKO MATSUBARA, JUNKO HARAGA, CHIKAKO OGAWA and HISASHI MASUYAMA \\ Department of Obstetrics and Gynecology, Okayama University Graduate School of Medicine, \\ Dentistry and Pharmaceutical Sciences, Okayama, Okayama 700-8558, Japan
}

Received May 14, 2019; Accepted January 2, 2020

DOI: $10.3892 /$ mco.2020.1989

\begin{abstract}
The aim of the present study was to determine whether chronic diseases (CD), such as hypertension, diabetes mellitus, dyslipidemia, heart diseases and cerebrovascular diseases, are occurrence risk factors and affect the survival of patients with gynecological cancers (GC). The correlations between $\mathrm{CD}$ and the characteristics and survival of 1,590 GC patients [685 with cervical cancer (CC), 613 with endometrial cancer (EM) and 292 with ovarian cancer (OV)] were investigated in the present study. Of the CD patients, 189 had CC (27.6\%), 265 had EM (43.2\%) and 72 had OV (24.7\%). The incidence of CD increased with age in GC patients. The number of $C D$ patients aged $\geq 70$ years, was 8.6-fold higher in the $C C$ group, 3.0-fold higher in the EM group, and 9.6-fold higher in the OV group compared with those aged $<50$ years. CD and excess body weight were associated with $\mathrm{GC}$ regardless of patient age. However, there was no correlation between $\mathrm{CD}$ and survival at any age in GC patients. These findings indicate that CD contribute to $>24 \%$ of the occurrence risk factors in GC patients in Japan.
\end{abstract}

\section{Introduction}

The incidence of gynecological cancers (GC) has increased in Japan, with an estimated 30,964 newly diagnosed patients in 2009. In recent years, the incidence of GC has increased among younger patients, as well as among older patients (1). The risk factors for GC differ by organ. For example, the development of invasive cervical cancer (CC) requires persistent infection by human papillomavirus $(2,3)$. Several occurrence risk factors for endometrial cancer (EM) have been established, including

Correspondence to: Dr Keiichiro Nakamura, Department of Obstetrics and Gynecology, Okayama University Graduate School of Medicine, Dentistry and Pharmaceutical Sciences, 2-5-1 Shikata-cho, Kita-ku, Okayama, Okayama 700-8558, Japan

E-mail: k-nakamu@cc.okayama-u.ac.jp

Key words: gynecological cancer, chronic diseases, occurrence risk factors excess body weight (4) and diabetes mellitus (DM) (5). The occurrence risk factors for ovarian cancer (OV) include age at diagnosis, family history of $\mathrm{OV}$, infertility treatment and assisted fertilization, obesity and metabolic syndromes (6). Recently, metabolic conditions, such as obesity, hyperlipidemia and DM, have been attracting increasing attention with respect to $\mathrm{OV}$ incidence $(7,8)$. Therefore, the onset of EM and OV appears to be associated with lifestyle and behavioral factors, such as dietary habits, physical activity, smoking and alcohol consumption.

Chronic diseases (CD) and cancer share common risk factors, including aging and unhealthy habits, such as smoking, poor diet, sedentary lifestyle, obesity and alcohol intake. CD include hypertension (HT), DM, dyslipidemia (DL), heart disease (HD) and cerebrovascular disease (CVD), and constitute $>20 \%$ of the occurrence risk factors for various cancers (9). In 2017, the Japanese Ministry of Health, Labour and Welfare reported that 108,000 CD patients were aged $<35$ years, 3,141,000 CD patients were aged 35-64 years, and $11,458,000 \mathrm{CD}$ patients were aged $\geq 65$ years among women without cancer in Japan (10).

The development rates of $\mathrm{CD}$ are rapidly increased by excess body weight (11), but there are no published details of the effect of CD on GC. Therefore, the aim of the present study was to investigate the correlations between $\mathrm{CD}$ and $\mathrm{GC}$, including CC.

\section{Patients and methods}

Study population. The present retrospective study reviewed the medical records of 1,590 GC patients who were treated at the Department of Obstetrics and Gynecology of Okayama University Hospital (Okayama, Japan) between April 2004 and December 2017. The study protocol was approved by the Institutional Review Board of Okayama University Hospital (1904-05). Several studies reported that the threshold for lowest risk of all-cause mortality was $\sim 100 \mathrm{~g}$ alcohol/week and $<10$ cigarettes/day $(12,13)$. All patients underwent a review of their medical history and lifestyle habits (smoking-positive: Current smokers of $\geq 10$ cigarettes/day; alcohol intake-positive: Alcohol intake of $\geq 14 \mathrm{~g} / \mathrm{day}$ ), physical examination and routine clinical staging. The patients were treated according to the Japan Society of Gynecologic Oncology clinical 
Table I. Incidence of GC among patients with chronic diseases in different age groups.

\begin{tabular}{|c|c|c|c|c|c|c|}
\hline \multirow[b]{2}{*}{ Chronic diseases } & \multicolumn{2}{|c|}{$\mathrm{CC}$} & \multicolumn{2}{|c|}{ EM } & \multicolumn{2}{|c|}{$\mathrm{OV}$} \\
\hline & Number & $(\%)$ & Number & $(\%)$ & Number & $(\%)$ \\
\hline \multicolumn{7}{|l|}{ HT, age (years) } \\
\hline$<50$ & 9 & 3.2 & 12 & 10 & 1 & 1.3 \\
\hline $50-59$ & 16 & 11.6 & 34 & 17 & 13 & 14.9 \\
\hline $60-69$ & 43 & 32.3 & 61 & 48.8 & 19 & 23.2 \\
\hline$\geq 70$ & 57 & 41.6 & 67 & 39.9 & 13 & 28.9 \\
\hline \multicolumn{7}{|l|}{ DM, age (years) } \\
\hline$<50$ & 4 & 1.4 & 13 & 10.8 & 2 & 2.6 \\
\hline $50-59$ & 10 & 7.2 & 17 & 8.5 & 3 & 3.4 \\
\hline $60-69$ & 15 & 11.2 & 20 & 16 & 4 & 4.9 \\
\hline$\geq 70$ & 12 & 8.8 & 31 & 18.5 & 6 & 13.3 \\
\hline \multicolumn{7}{|l|}{ DL, age (years) } \\
\hline$<50$ & 2 & 0.7 & 5 & 4.2 & 0 & 0 \\
\hline $50-59$ & 4 & 2.9 & 18 & 9 & 8 & 9.2 \\
\hline $60-69$ & 14 & 10.5 & 27 & 21.6 & 8 & 9.8 \\
\hline$\geq 70$ & 14 & 10.2 & 42 & 25 & 7 & 15.6 \\
\hline \multicolumn{7}{|l|}{ HD, age (years) } \\
\hline$<50$ & 2 & 0.7 & 4 & 3.3 & 1 & 1.3 \\
\hline $50-59$ & 2 & 1.4 & 6 & 3 & 2 & 2.3 \\
\hline $60-69$ & 4 & 3 & 13 & 10.4 & 1 & 1.2 \\
\hline$\geq 70$ & 15 & 10.9 & 8 & 4.8 & 2 & 4.4 \\
\hline \multicolumn{7}{|l|}{ CVD, age (years) } \\
\hline$<50$ & 3 & 1.1 & 0 & 0 & 0 & 0 \\
\hline $50-59$ & 2 & 1.4 & 5 & 2.5 & 0 & 0 \\
\hline $60-69$ & 6 & 4.5 & 6 & 4.8 & 3 & 3.7 \\
\hline$\geq 70$ & 13 & 9.5 & 6 & 3.6 & 3 & 6.7 \\
\hline
\end{tabular}

GC, gynecological cancers; CC, cervical cancer; EM, endometrial cancer; OV, ovarian cancer; HT, hypertension; DM, diabetes mellitus; DL, dyslipidemia; HD, heart diseases; CVD, cerebrovascular diseases.

guidelines (14-16). The treatment options for gynecological cancer included surgery, radiotherapy and/or chemotherapy, depending on tumor stage and additional risk factors.

Data collection. The patients were asked to complete questionnaires on their history of cardiac disease, lifestyle habits (smoking and alcohol intake), and medications for HT, DM and DL. The measurements included standard medical examinations, such as height, weight, blood pressure, fasting blood glucose, hemoglobin A1c and serum lipid profile, including triglyceride (TG), serum high-density lipoprotein cholesterol (HDL-C) and low-density lipoprotein cholesterol (LDL-C) levels. Patients with HT were defined as those with blood pressure $\geq 140 / 90 \mathrm{mmHg}$, or those on antihypertensive medication. Patients with DM were defined as those with hemoglobin A1c $\geq 6.5 \%$, or those receiving antidiabetic medication. Patients with DL were determined as those with TG $\geq 150 \mathrm{mg} / \mathrm{dl}$, HDL-C $<40 \mathrm{mg} / \mathrm{dl}$, and LDL-C $\geq 140 \mathrm{mg} / \mathrm{dl}$, or those receiving medication for DL. The presence of HD and CVD were assessed based on self-reports. Height and weight were measured on admission, prior to any therapeutic intervention. Body mass index (BMI) was defined according to the 2015 World Health Organization classification as follows: Underweight, $<18.5 \mathrm{~kg} / \mathrm{m}^{2}$; normal weight, $18.5-24.99 \mathrm{~kg} / \mathrm{m}^{2}$; overweight, $25.0-29.99 \mathrm{~kg} / \mathrm{m}^{2}$; and obese, $\geq 30.0 \mathrm{~kg} / \mathrm{m}^{2}$.

Statistical analysis. Data were analyzed using $\chi^{2}$ and Mann-Whitney U-tests for comparisons, and by one-way analysis of variance followed by Fisher's protected least significant difference test for all pairwise comparisons. Survival curves were constructed using the Kaplan-Meier method, and differences between survival curves were examined using the log-rank test. Analyses were performed using SPSS software, version 23.0 (IBM Corp.), with the significance level set at 0.05 .

\section{Results}

Patient characteristics. CC, EM and OV patients were aged 25-92, 23-91 and 15-84 years, respectively (median CC age, 55.0 years; median EM age, 59.0 years; and median OC age, 57.0 years). Age was divided into four groups: $<50,50-59$, 
Table II. Baseline characteristics and CD in patients with gynecological cancers.

\begin{tabular}{|c|c|c|c|c|c|c|c|c|c|}
\hline \multirow{2}{*}{$\begin{array}{l}\text { Baseline } \\
\text { characteristics }\end{array}$} & \multicolumn{3}{|c|}{$\mathrm{CC}$} & \multicolumn{3}{|c|}{ EM } & \multicolumn{3}{|c|}{$\mathrm{OV}$} \\
\hline & Number & $(\%)$ & P-value & Number & $(\%)$ & $\mathrm{P}$-value & Number & $(\%)$ & P-value \\
\hline Stage & & & - & & & $<0.001^{\mathrm{a}}$ & & & $<0.001^{\mathrm{a}}$ \\
\hline I, II & 437 & 63.8 & & 460 & 75.0 & & 139 & 47.6 & \\
\hline III, IV & 248 & 36.2 & & 153 & 25.0 & & 153 & 52.4 & \\
\hline HT & & & - & & & $<0.001^{\mathrm{a}}$ & & & 0.375 \\
\hline Present & 125 & 18.2 & & 174 & 28.4 & & 46 & 15.8 & \\
\hline Absent & 560 & 81.8 & & 439 & 71.6 & & 246 & 84.2 & \\
\hline DM & & & - & & & $<0.001^{\mathrm{a}}$ & & & 0.601 \\
\hline Present & 41 & 6 & & 81 & 13.2 & & 15 & 5.1 & \\
\hline Absent & 644 & 94 & & 532 & 86.8 & & 277 & 94.9 & \\
\hline DL & & & - & & & $<0.001^{\mathrm{a}}$ & & & 0.075 \\
\hline Present & 34 & 5 & & 92 & 15.0 & & 23 & 7.9 & \\
\hline Absent & 651 & 95 & & 521 & 85.0 & & 269 & 92.1 & \\
\hline HD & & & - & & & 0.126 & & & 0.272 \\
\hline Present & 23 & 3.4 & & 31 & 5.1 & & 6 & 2.1 & \\
\hline Absent & 662 & 96.6 & & 582 & 94.9 & & 286 & 97.9 & \\
\hline CVD & & & - & & & 0.453 & & & 0.229 \\
\hline Present & 24 & 3.5 & & 17 & 2.8 & & 6 & 2.1 & \\
\hline Absent & 661 & 96.5 & & 596 & 97.2 & & 286 & 97.9 & \\
\hline $\mathrm{BMI}, \mathrm{kg} / \mathrm{m}^{2}$ & & & - & & & $<0.001^{\mathrm{a}}$ & & & 0.537 \\
\hline$<25.0$ & 549 & 80.1 & & 370 & 60.4 & & 239 & 81.8 & \\
\hline$\geq 25.0$ & 136 & 19.9 & & 243 & 39.6 & & 53 & 18.2 & \\
\hline Smoking & & & - & & & $<0.001^{\mathrm{a}}$ & & & $<0.001^{\mathrm{a}}$ \\
\hline Negative & 524 & 76.5 & & 577 & 94.1 & & 274 & 93.8 & \\
\hline Positive & 161 & 23.5 & & 36 & 5.9 & & 18 & 6.2 & \\
\hline Alcohol & & & - & & & $<0.001^{\mathrm{a}}$ & & & $0.026^{\mathrm{a}}$ \\
\hline Negative & 609 & 88.9 & & 580 & 94.6 & & 273 & 93.5 & \\
\hline Positive & 76 & 11.1 & & 33 & 5.4 & & 19 & 6.5 & \\
\hline
\end{tabular}

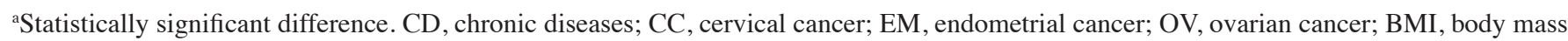
index, HT, hypertension; DM, diabetes mellitus; DL, dyslipidemia; HD, heart diseases; CVD, cerebrovascular diseases.

$60-69$ and $\geq 70$ years. The $<50$ years group included $277 \mathrm{CC}$ (40.4\%), 120 EM (19.6\%) and 78 OV (26.7\%) patients; the $50-59$ years group included 138 CC (20.2\%), 200 EM (32.6\%) and $87 \mathrm{OV}(29.8 \%)$ patients; the 60-69 years group included 133 CC (19.4\%), 168 EM (27.4\%) and 82 OV (28.1\%) patients; and the $\geq 70$ years group included $137 \mathrm{CC}(20.0 \%), 125 \mathrm{EM}$ (20.4\%) and 45 OV (15.4\%) patients.

The HT, DM, DL, HD and CVD patients in the $<50$ years group included $9,4,2,2$ and $3 \mathrm{CC}$ patients, respectively (3.2, 1.4, 0.7, 0.7 and 1.1\%, respectively); $12,13,5,4$ and 0 EM patients, respectively $(10.0,10.8,4.2,3.3$ and $0 \%$, respectively); and 1, 2, 0, 1 and 0 OV patients, respectively (1.3, 2.6, $0,1.3$ and $0 \%$, respectively). The HT, DM, DL, HD and CVD patients in the 50-59 years group included 16, 10, 4, 2 and 2 CC patients, respectively $(11.6,7.2,2.9,1.4$ and $1.4 \%$, respectively); 34, 17, 18, 6 and 5 EM patients, respectively (17.0, 8.5, 9.0, 3.0 and $2.5 \%$, respectively); and $13,3,8,2$ and $0 \mathrm{OV}$ patients, respectively $(14.9,3.4,9.2,2.3$ and $0 \%$, respectively).

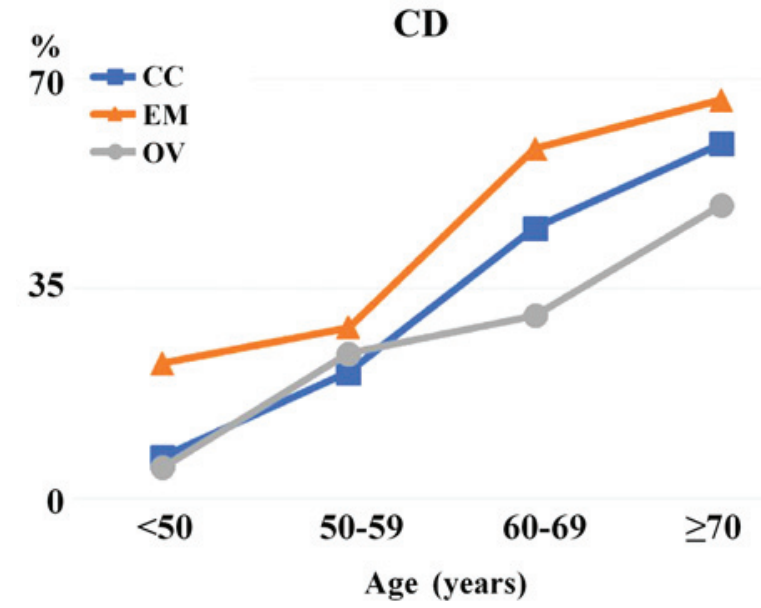

Figure 1. The percentage of chronic diseases (CD) in cervical cancer (CC), endometrial cancer (EM) and ovarian cancer (OV) patients was examined in the four age groups: $<50,50-59,60-69$ and $\geq 70$ years. 


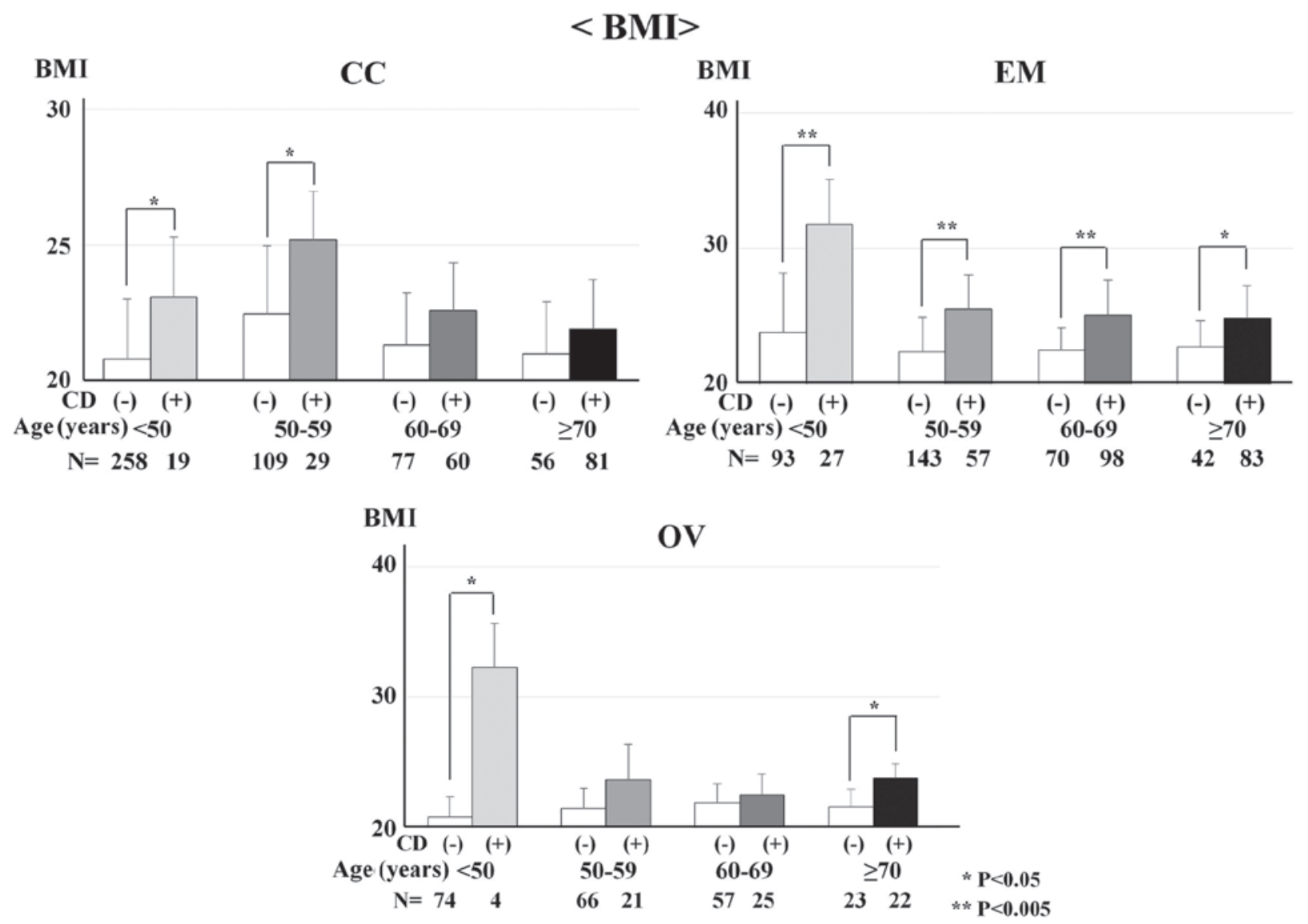

Figure 2. The association of body mass index (BMI) with chronic diseases (CD) and age (four groups: $<50,50-59,60-69$ and $\geq 70$ years) was examined in cervical cancer (CC), endometrial cancer (EM) and ovarian cancer $(\mathrm{OV})$ patients. ${ }^{*} \mathrm{P}<0.05 ;{ }^{* *} \mathrm{P}<0.005$.

The HT, DM, DL, HD and CVD patients in the 60-69 years group included 43, 15, 14, 4 and 6 CC patients, respectively $(32.3,11.2,10.5,3.0$ and $4.5 \%$, respectively); $61,20,27,13$ and 6 EM patients, respectively $(48.8,16.0,21.6,10.4$ and $4.8 \%$, respectively); and 19, 4, 8, 1 and 3 OV patients, respectively (23.2, 4.9, 9.8, 1.2 and $3.7 \%$, respectively). Finally, the HT, DM, DL, HD and CVD patients in the $\geq 70$ years group included $57,12,14,15$ and $13 \mathrm{CC}$ patients, respectively $(41.6,8.8,10.2$, 10.9 and $9.5 \%$, respectively); 67, 31, 42, 8 and 6 EM patients, respectively (39.9, 18.5, 25.0, 4.8 and $3.6 \%$, respectively); and $13,6,7,2$ and 3 OV patients, respectively (28.9, 13.3, 15.6, 4.4 and $6.7 \%$, respectively) (Table I).

The associations of each GC type [CC $(n=685)$, EM $(n=613)$ and OV $(n=292)]$ with clinical characteristics (cancer stage, HT, DM, DL, HD, CVD, BMI, smoking and alcohol intake) were assessed (Table II). Regarding FIGO stage, there were significantly more EM patients with early-stage disease compared with $\mathrm{CC}$ patients $(\mathrm{P}<0.001)$. Conversely, the number of advanced-stage OV patients was significantly higher compared with $\mathrm{CC}$ patients $(\mathrm{P}<0.001)$. The incidence of HT, DM, DL and high BMI were significantly higher in EM patients compared with CC patients (all $\mathrm{P}<0.001$ ). Furthermore, significantly more CC patients were smoking- and alcohol intake-positive compared with EM and OV patients $(\mathrm{P}<0.001$, $\mathrm{P}<0.001, \mathrm{P}<0.001$ and $\mathrm{P}=0.026$, respectively).

The effect of $C D$ was examined by determining how many of CC, EM and OV patients in each of the four age groups had CD. CD patients were divided into 189 CC (27.6\%), 265 EM
(43.2\%) and $72 \mathrm{OV}(24.7 \%)$ patients. Patients in the $<50$ years group with CD included $19 \mathrm{CC}(6.9 \%), 27 \mathrm{EM}(22.5 \%)$ and 4 OV (5.1\%) patients. CD patients in the 50-59 years group included 29 CC (21.0\%), 57 EM (28.5\%) and 21 OV (24.1\%) patients. CD patients in the 60-69 years group included $60 \mathrm{CC}$ (45.1\%), 98 EM (58.3\%), and 25 OV (30.5\%) patients. Finally, CD patients in the $\geq 70$ years group included $81 \mathrm{CC}(59.1 \%)$, 83 EM (66.4\%) and 22 OV (48.9\%) patients. CC patients aged $\geq 70$ years and EM patients aged $60-69$ and $\geq 70$ years accounted for $>50 \%$ of those with CD. Therefore, the number of patients with $\mathrm{CD}$ aged $\geq 70$ years was 8.6-fold higher in the CC group, 3.0-fold higher in the EM group, and 9.6-fold higher in the $\mathrm{OV}$ group compared with patients aged $<50$ years (Fig. 1).

CD was examined for its association with BMI, smoking and alcohol intake at any age in CC, EM and OV patients. $\mathrm{BMI}$ and $\mathrm{CD}$ were significantly associated in $\mathrm{CC}$ patients aged $<50$ and 50-59 years $(\mathrm{P}=0.025$ and $\mathrm{P}=0.010$, respectively). $\mathrm{BMI}$ and $\mathrm{CD}$ were significantly associated in all age groups in $\mathrm{EM}$ patients $(\mathrm{P}<0.001, \mathrm{P}<0.001, \mathrm{P}<0.001$ and $\mathrm{P}=0.014$, respectively). BMI and $\mathrm{CD}$ were significantly associated in OV patients aged 50-59 and $\geq 70$ years $(\mathrm{P}=0.043$ and $\mathrm{P}=0.004$, respectively; Fig. 2). However, there was no association between $\mathrm{CD}$ and smoking or alcohol intake at any age in CC, EM or OV patients.

In the present study, the median overall survival (OS) rates for patients with CC, EM and OV were 44.0, 40.0 and 32.5 months, respectively. The follow-up period was 1-174, 

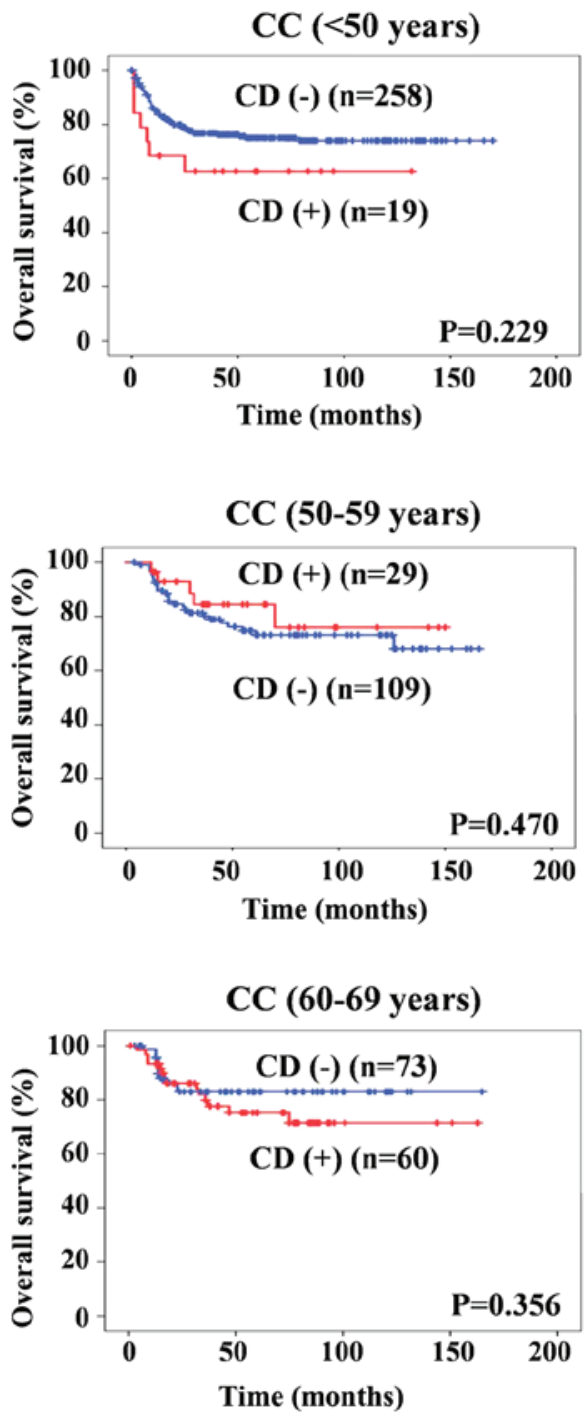

$\mathrm{CC}(\geq 70$ years $)$

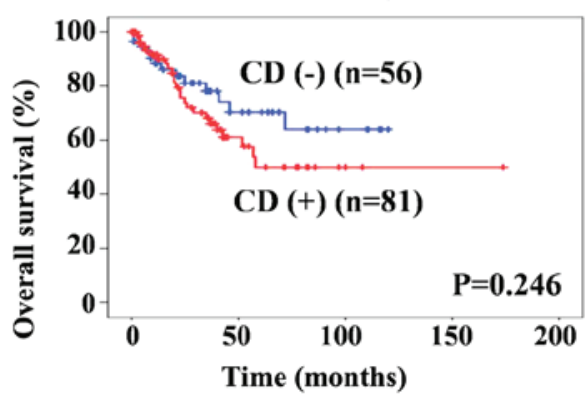

EM ( $<50$ years $)$

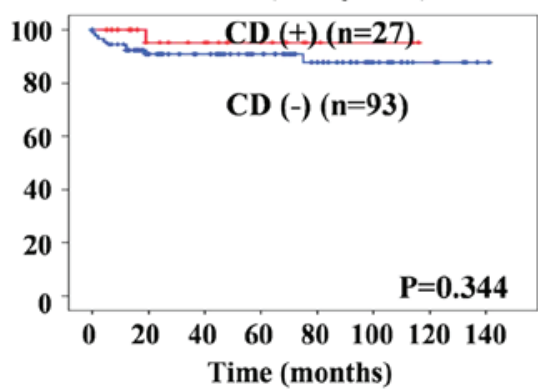

EM (50-59 years)

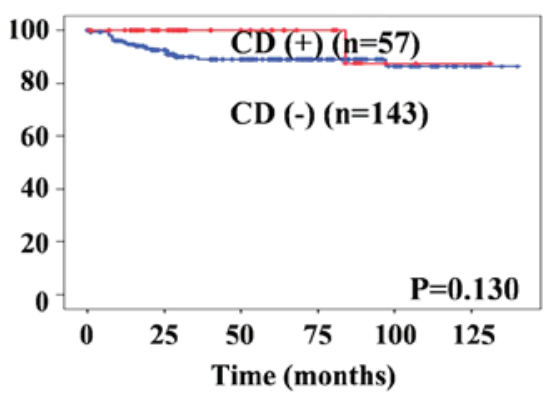

EM (60-69 years)

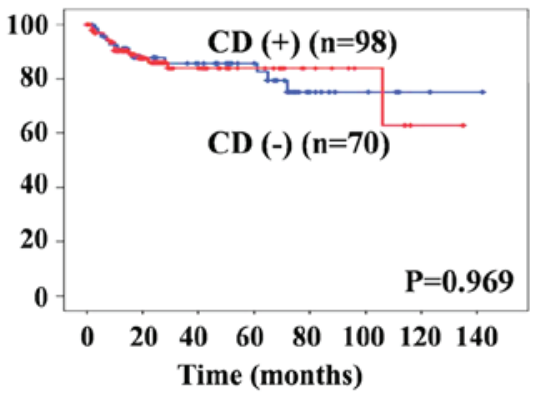

EM ( $\geq 70$ years $)$

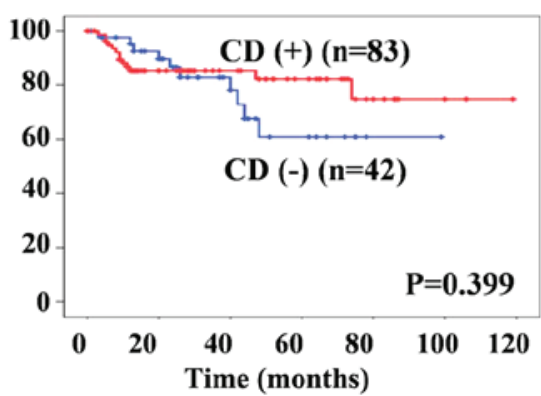

OV ( $<50$ years)

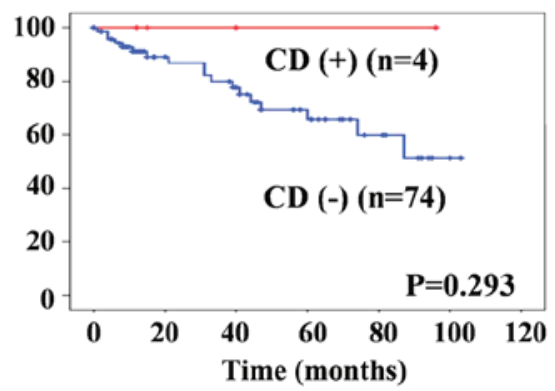

OV (50-59 years)

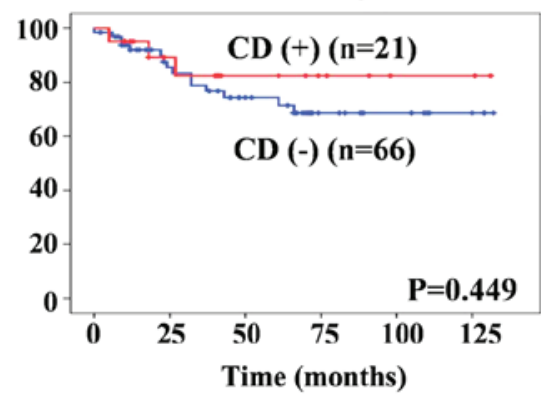

OV (60-69 years)

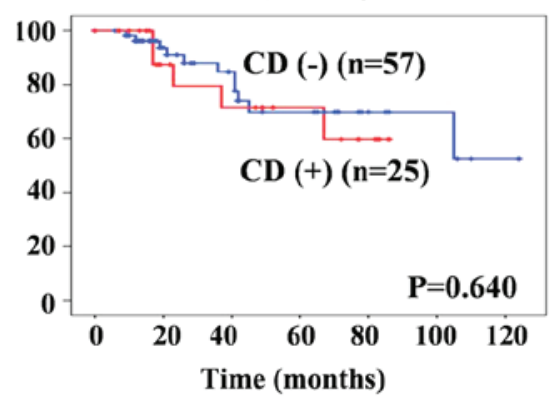

OV $(\geq 70$ years $)$

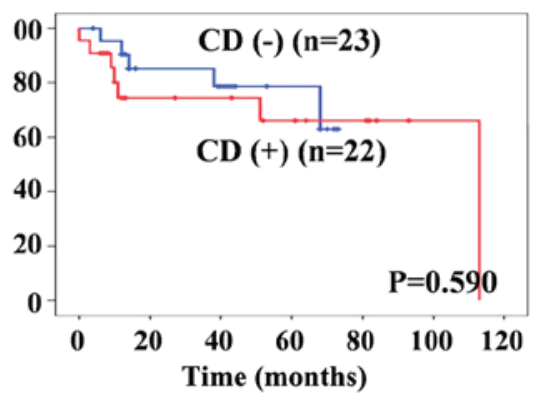

Figure 3. Kaplan-Meier curves for overall survival (OS) in relation to CD according to age (four groups: $<50,50-59,60-69$ and $\geq 70$ years) in cervical cancer (CC), endometrial cancer (EM) and ovarian cancer (OV) patients.

1-146 and 1-132 months, respectively. The OS curves for the 1,590 GC patients according to their CD are shown in Fig. 3. There was no significant correlation between $\mathrm{CD}$ and survival at any age in CC, EM or OV patients.

\section{Discussion}

The occurrence risk factors for cancer include smoking, an unhealthy diet, obesity, sedentary lifestyle, DM, HT and alcohol abuse, either alone or in combination. Accumulating evidence has suggested that obesity is an important occurrence risk factor for EM, and BMI is significantly associated with symptoms in EM patients (17). Zhang et al also demonstrated that DM is associated with EM (5), and several studies have reported the association of metabolic markers of obesity, including elevated blood glucose, TG and total cholesterol levels, with EM (18).

$\mathrm{CD}$ and cancer share common risk factors, particularly those associated with an unhealthy lifestyle, such as smoking, an unhealthy diet, physical inactivity, obesity and alcohol 
intake. CD is known to contribute to $>20 \%$ of occurrence risk factors for various cancers (9); however, to the best of our knowledge, this is the first study to describe an association between $\mathrm{CD}$ and $\mathrm{GC}$, including $\mathrm{CC}$.

In the present study, CD, including HT, DM, DL, HD and CVD, were examined in patients with CC, EM and OV. For all diseases, we observed a high frequency of EM, CC and OV, in decreasing order. For example, HT was observed in 18.2, 28.4 and $15.8 \%$ of CC, EM and OV patients, respectively; DL was observed in 5.0, 15.0 and 7.9\% of CC, EM and OV patients, respectively; DM occurred in 6.0, 13.2 and 5.1\% of CC, EM and $\mathrm{OV}$ patients, respectively; HD was diagnosed in 3.4, 5.1 and $2.1 \%$ of CC, EM and OV patients, respectively; and CVD was recorded in 3.5, 2.8 and $2.1 \%$ of $\mathrm{CC}$, EM and OV patients, respectively. Among the $\mathrm{CD}$ patients, 27.6, 43.2 and $24.7 \%$ had CC, EM and OV, respectively. The incidence of CD was found to increase with age in GC patients, with $\mathrm{CC}$ patients aged $>70$ years and EM patients aged $>60$ years accounting for $>50 \%$ of patients with CD. Moreover, the numbers of any disease increased with increasing age, regardless of the number of CD.

CD were examined for their association with lifestyle factors, such as obesity, smoking and alcohol intake, at any age in all GC patients. In the present study, $\mathrm{CD}$ were more prevalent among EM patients compared with $\mathrm{CC}$ and $\mathrm{OV}$ patients. Among the CD patients, 27.6, 43.2 and 24.7\% had CC, EM and $\mathrm{OV}$, respectively. Of note, occurrence risk factors for EM have been established, including HT, DM and DL.

However, there was no association of smoking and alcohol intake with $\mathrm{CD}$. We also determined whether $\mathrm{CD}$ were associated with outcome in GC patients, and found that the presence of $\mathrm{CD}$ was not a prognostic predictor for $\mathrm{CC}, \mathrm{EM}$ or $\mathrm{OV}$ patients.

There were certain limitations to the present study. The number of patients was relatively small, and the examinations were performed at a single institution. Further prospective studies involving more patients and multiple institutions should provide more definitive data to verify the significance of our findings.

In conclusion, the presence of CD appears to contribute to $>24 \%$ of the occurrence risk factors for GC patients in Japan.

\section{Acknowledgements}

The authors would like to thank all those who contributed to the present study, particularly the statisticians and colleagues of Okayama University Graduate School of Medicine, Dentistry and Pharmaceutical Sciences. We appreciate their help with data management and statistical support. We would also like to thank Sarah Williams, PhD, and H. Nikki March, $\mathrm{PhD}$ for editing a draft of this manuscript.

\section{Funding}

No funding was received.

\section{Availability of data and materials}

The datasets used and/or analyzed during the present study are available from the corresponding author on reasonable request.

\section{Authors' contributions}

$\mathrm{KN}$ and $\mathrm{KO}$ contributed to the conception, design and conduction of the study, and analysis and interpretation of the data. HM, YM, JH, CO and HM contributed to data collection and the conduction of the study. All the authors have read and approved the final version of this manuscript.

\section{Ethics approval and consent to participate}

The present study was performed according to the principles set out in the Declaration of Helsinki 1964 and all subsequent revisions, and was approved by the Institutional Review Board of Okayama University Hospital (IRB approval no. 1904-005).

\section{Patient consent for publication}

Not applicable.

\section{Competing interests}

All the authors declare that they have no competing interests to disclose.

\section{References}

1. Hori M, Matsuda T, Shibata A, Katanoda K, Sobue T and Nishimoto H; Japan Cancer Surveillance Research Group: Cancer incidence and incidence rates in Japan in 2009: A study of 32 population-based cancer registries for the monitoring of cancer incidence in Japan (MCIJ) project. Jpn J Clin Oncol 45: 884-891, 2015.

2. de Sanjose S, Quint WG, Alemany L, Geraets DT Klaustermeier JE, Lloveras B, Tous S, Felix A, Bravo LE, Shin HR, et al: Human papillomavirus genotype attribution in invasive cervical cancer: A retrospective cross-sectional worldwide study. Lancet Oncol 11: 1048-1056, 2010.

3. Al Moustafa AE, Ghabreau L, Akil N, Rastam S, Alachkar A and Yasmeen A: High-risk HPVs and human carcinomas in the Syrian population. Front Oncol 4: 68, 2014.

4. Aune D, Navarro Rosenblatt DA, Chan DS, Vingeliene S, Abar L, Vieira AR, Greenwood DC, Bandera EV and Norat T: Anthropometric factors and endometrial cancer risk: A systematic review and dose-response meta-analysis of prospective studies. Ann Oncol 26: 1635-1648, 2015.

5. Zhang Y, Liu Z, Yu X, Zhang X, Lü S, Chen X and Lü B: The association between metabolic abnormality and endometrial cancer: A large case-control study in China. Gynecol Oncol 117: 41-46, 2010.

6. Xie H, Hou Y, Cheng J, Openkova MS, Xia B, Wang W, Li A, Yang K, Li J, Xu H, et al: Metabolic profiling and novel plasma biomarkers for predicting survival in epithelial ovarian cancer. Oncotarget 8: 32134-32146, 2017.

7. Chen Y, Zhang L, Liu W and Wang K: Case-control study of metabolic syndrome and ovarian cancer in Chinese population. Nutr Metab (Lond) 14: 21, 2017.

8. Nagle CM, Dixon SC, Jensen A, Kjaer SK, Modugno F, deFazio A, Fereday S, Hung J, Johnatty SE; Australian Ovarian Cancer Study Group, et al: Obesity and survival among women with ovarian cancer: Results from the Ovarian cancer association consortium. Br J Cancer 113: 817-826, 2015.

9. Tu H, Wen CP, Tsai SP, Chow WH, Wen C, Ye Y, Zhao H, Tsai MK, Huang M, Dinney CP, et al: Cancer risk associated with chronic diseases and disease markers: Prospective cohort study. BMJ 360: k134, 2018.

10. Ministry of Health labor and Welfare reports. Patient survey overview in 2017 year in Japan, pp16-32, 2017 (Japanese).

11. Chen Y, Copeland WK, Vedanthan R, Grant E, Lee JE, Gu D, Gupta PC, Ramadas K, Inoue M, Tsugane S, et al: Association between body mass index and cardiovascular disease mortality in east Asians and south Asians: Pooled analysis of prospective data from the Asia Cohort Consortium. BMJ 347: f5446, 2013. 
12. Wood AM, Kaptoge S, Butterworth AS, Willeit P, Warnakula S, Bolton T, Paige E, Paul DS, Sweeting M, Burgess S, et al: Risk thresholds for alcohol consumption: Combined analysis of individual-participant data for 599912 current drinkers in 83 prospective studies. Lancet 391: 1513-1523, 2018.

13. Whitfield JB, Heath AC, Madden PAF, Landers JG and Martin NG: Effects of high alcohol intake, alcohol-related symptoms and smoking on mortality. Addiction 113: 158-166, 2018.

14. Ebina Y, Mikami M, Nagase S, Tabata T, Kaneuchi M, Tashiro H, Mandai M, Enomoto T, Kobayashi Y, Katabuchi H, et al: Japan Society of Gynecologic Oncology guidelines 2017 for the treatment of uterine cervical cancer. Int J Clin Oncol 24: 1-19, 2019.

15. Ebina Y, Katabuchi H, Mikami M, Nagase S, Yaegashi N, Udagawa Y, Kato H, Kubushiro K, Takamatsu K, Ino K and Yoshikawa H: Japan Society of Gynecologic Oncology guidelines 2013 for the treatment of uterine body neoplasms. Int J Clin Oncol 21: 419-434, 2016.
16. Komiyama S, Katabuchi H, Mikami M, Nagase S, Okamoto A, Ito K, Morishige K, Suzuki N, Kaneuchi M, Yaegashi N, et al: Japan Society of Gynecologic Oncology guidelines 2015 for the treatment of ovarian cancer including primary peritoneal cancer and fallopian tube cancer. Int J Clin Oncol 21: 435-446, 2016.

17. Jenabi E and Poorolajal J: The effect of body mass index on endometrial cancer: A meta-analysis. Public Health 129: 872-880, 2015.

18. Friedenreich CM, Biel RK, Lau DC, Csizmadi I, Courneya KS, Magliocco AM, Yasui Y and Cook LS: Case-control study of the metabolic syndrome and metabolic risk factors for endometrial cancer. Cancer Epidemiol Biomarkers Prev 20: 2384-2395, 2011.

(i) $9 \odot$ This work is licensed under a Creative Commons Attribution-NonCommercial-NoDerivatives 4.0 International (CC BY-NC-ND 4.0) License. 